\title{
Performance Guarantees for Multiobjective Model Predictive Control*
}

\author{
Lars Grüne and Marleen Stieler
}

\begin{abstract}
We consider model predictive control with terminal conditions for discrete-time multiobjective optimal control problems for classical and economic stage costs. In this paper it is demonstrated that choosing an appropriate solution to the multiobjective optimization problem in each step of the MPC algorithm yields a near Pareto-optimal infinite-horizon performance of the MPC feedback for each cost criterion.
\end{abstract}

\section{INTRODUCTION}

Multiobjective (MO) model predictive control (MPC) is an attempt to incorporate optimal control problems with multiple objectives into the MPC framework. A multiobjective optimal control problem typically arises if one system/process has multiple (conflicting) objectives or if several agents with individual dynamics all have their own objective(s) but are coupled through inputs, dynamics, and/or costs. The existing research on multiobjective model predictive control is usually embedded in a cooperative setting and aims to obtain stability. A common approach is to define a weighted sum of all objectives, see e.g. [2], [6], [14], [16]. Since this approach reduces MO MPC to classical scalar-valued MPC, stability can be achieved by proving that the weighted sum of objectives is a Lyapunov function. Advantageous features of this strategy are the low computiational effort and that all the results from classical MPC are readily available. Other approaches to handle the MO optimization problem are hierarchical MPC algorithms (e.g. [9]), the so called utopiatracking approach in [18] and iterative schemes, see [11]. The aforementioned all prove stability via the construction of a Lyapunov function. Conceptually closer to our work are the refs. [5], [10], [12] as well as [13], [17]. In the former papers in each step of the MO MPC algorithm an approximation to the Pareto front is calculated and then - according to some pre-specified criterion - a Pareto optimum is chosen. The latter skip the approximation of the Pareto front.

Though often possible, a performance analysis is usually not carried out for MO MPC. An exception is the work [6], making a performace statement on the (unweighted) sum of objectives. While one could extend this result by deriving estimates for the weighted sum of objectives or for the most prioritized objective, in this paper we pursue a different approach and present an MO MPC scheme with terminal conditions which enables us to make performance statements for every single objective. One reason why such an analysis is needed is that statements on the performance of the weighted sum do not imply statements on the single

*Supported by DFG Grant Gr1569/13-1.

L. Grüne and M. Stieler are with the Department of Mathematics, University of Bayreuth, 95440 Bayreuth, Germany. \{lars.gruene, marleen.stieler\}@uni-bayreuth.de objectives, see [8]. The setting of multiple objectives for one system or multiple systems with own objective can both be handled. The idea of our approach is the following: In each iteration of the MPC algorithm we choose a Pareto optimal sequence within certain constraints and - as usual in MPC - apply the first piece of it to the system. We will show that the infinite-horizon performance of our MO MPC algorithm is bounded by the objective of a Pareto optimum on a finite horizon for each objective.

The novelty of our findings mainly arises from the ability to make a statement on the performance of every objective and from the fact that, a priori, we do not have to define a weighting or prioritization of objectives. Moreover, we do not restrict ourselves to a specific technique to calculate the Pareto optima. Hence, our theoretical analysis does not depend on such a technique, and we can choose the optimization method that suits best for the given problem.

\section{Setting And Definitions}

We consider a discrete-time system

$$
x^{+}=f(x, u), f: \mathbb{R}^{n} \times \mathbb{R}^{m} \rightarrow \mathbb{R}^{n}
$$

and admissible state and control spaces $\mathbb{X} \subseteq \mathbb{R}^{n}$ and $\mathbb{U} \subseteq$ $\mathbb{R}^{m}$. Moreover, stage costs $\ell_{i}: \mathbb{X} \times \mathbb{U} \rightarrow \mathbb{R}_{\geq 0}$ and terminal costs $F_{i}: \mathbb{X}_{0} \rightarrow \mathbb{R}, i \in\{1, \ldots, s\}$, are given and we define the cost functionals

$$
J_{i}^{N}(x, \mathbf{u}):=\sum_{k=0}^{N-1} \ell_{i}(x(k), u(k))+F_{i}(x(N)),
$$

that we aim to minimize wrt. $\mathbf{u}$. Here, the expression $x(\cdot)$ refers to the trajectory corresponding to the control sequence $\mathbf{u}=(u(0), u(1), \ldots, u(N-1)) \in \mathbb{U}^{N}$ and initial value $x$. If necessary, we will use the notation $x^{\mathbf{u}}(\cdot, x)$ to indicate the control sequence and the initial value generating the respective trajectory.

Our setting can reflect different situations. Either (1) is one system with multiple objectives to be minimized, or (1) is a collection of individual systems

$$
x^{+}=\left(\begin{array}{c}
x_{1}^{+} \\
\vdots \\
x_{p}^{+}
\end{array}\right)=\left(\begin{array}{c}
f_{1}(x, u) \\
\vdots \\
f_{p}(x, u)
\end{array}\right)=: f(x, u),
$$

with $f_{i}: \mathbb{R}^{n} \times \mathbb{R}^{m} \rightarrow \mathbb{R}^{n_{i}}$ and $n=\sum_{i=1}^{p} n_{i}, x_{i} \in \mathbb{R}^{n_{i}}$, where each system has at least one cost criterion $\ell_{i}$ (i.e. $s \geq p$ ). In an MPC approach with terminal constraints we 
have to solve the problem

$$
\begin{array}{ll} 
& \min _{\mathbf{u}} \underbrace{\left(J_{1}^{N}(x, \mathbf{u}), \ldots, J_{s}^{N}(x, \mathbf{u})\right)}_{=: J^{N}(x, \mathbf{u})} \\
\text { s.t. } & x(k+1)=f(x(k), u(k)), \quad k=0, \ldots, N-1, \\
& x(k) \in \mathbb{X}, \quad k=1, \ldots, N-1, \\
& x(N) \in \mathbb{X}_{0} \subseteq \mathbb{X}, \\
& \mathbf{u} \in \mathbb{U}^{N} .
\end{array}
$$

in a sequential manner. Since the terminal constraint $x(N) \in$ $\mathbb{X}_{0}$ can generally not be satisfied by all initial values $x \in$ $\mathbb{X}$, we define the feasible set $\mathbb{X}_{N}:=\left\{x \in \mathbb{X} \mid \exists \mathbf{u} \in \mathbb{U}^{N}\right.$ : $\left.x(k) \in \mathbb{X}, k=1, \ldots, N-1, x(N) \in \mathbb{X}_{0}\right\}$, cf. [7, Definition 3.9] or [14, Section 2.3]. For $x \in \mathbb{X}_{N}$ we define the set of admissible controls for the Pareto optimization problem (4) by $\mathbb{U}^{N}(x):=\left\{\mathbf{u} \in \mathbb{U}^{N} \mid x(k+1)=f(x(k), u(k)), k=\right.$ $\left.0, \ldots, N-1, x(k) \in \mathbb{X}, k=1, \ldots, N-1, x(N) \in \mathbb{X}_{0}\right\}$.

Due to the fact that (4) contains more than one cost functional, in general it is not possible to find an admissible control sequence $\mathbf{u}$ that minimizes all cost functionals simultaneously, giving rise to the following cooperative notion of optimality.

Definition 1 (Pareto Optimality, Nondominated Point): A control sequence $\mathbf{u}^{\star} \in \mathbb{U}^{N}(x)$ is a Pareto optimal (control) sequence (POS) of length $N$ for initial value $x \in \mathbb{X}_{N}$ if there is no $\mathbf{u} \in \mathbb{U}^{N}(x)$ such that

$$
\begin{aligned}
& \forall i \in\{1, \ldots, s\}: J_{i}^{N}(x, \mathbf{u}) \leq J_{i}^{N}\left(x, \mathbf{u}^{\star}\right) \text { and } \\
& \exists i \in\{1, \ldots, s\}: J_{i}^{N}(x, \mathbf{u})<J_{i}^{N}\left(x, \mathbf{u}^{\star}\right) .
\end{aligned}
$$

The objective value $J^{N}\left(x, \mathbf{u}^{\star}\right)$ is called nondominated. The set of all POS of length $N$ for initial value $x \in \mathbb{X}_{N}$ will be denoted by $\mathbb{U}_{\mathcal{P}}^{N}(x)$.

Usually, Pareto optimal sequences are not unique. It is rather typical that there exists a set or even continuum of such sequences as shown in Fig. 1 for the case of two objectives. The

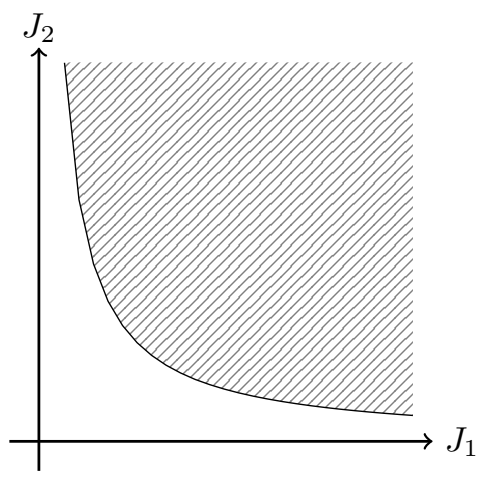

Fig. 1. Schematic illustration of a Pareto front for two objectives.

gray surface represent the set of admissible values $\mathcal{J}^{N}(x):=$ $\left\{J^{N}(x, \mathbf{u})=\left(J_{1}^{N}(x, \mathbf{u}), \ldots, J_{s}^{N}(x, \mathbf{u})\right) \mid \mathbf{u} \in \mathbb{U}^{N}(x)\right\}$, the black curve the set $\mathcal{J}_{\mathcal{P}}^{N}(x):=\left\{\left(J_{1}^{N}(x, \mathbf{u}), J_{2}^{N}(x, \mathbf{u})\right) \mid \mathbf{u} \in\right.$ $\left.\mathbb{U}_{\mathcal{P}}^{N}(x)\right\}$ of nondominated values. This set is often referred to as the efficient or nondominated set or Pareto front. Even though all points on the black curve are equally optimal in terms of the optimization problem (4), they are obviously not from each objective's point of view.

From the definition of Pareto optimal sequences we deduce the following property, which is similar to the Dynamic Programming Principle for single-objective optimization problems.

Lemma 2 (Tails of POS are POS): If $\mathbf{u}^{\star} \in \mathbb{U}_{\mathcal{P}}^{N}(x)$, then $\mathbf{u}^{\star, K}:=\mathbf{u}^{\star}(\cdot+K) \in \mathbb{U}_{\mathcal{P}}^{N-K}\left(x^{\mathbf{u}^{\star}}(K, x)\right)$ for all $K \in \mathbb{N}_{<N}$, where $\mathbf{u}^{\star}(\cdot+K):=\left(\mathbf{u}^{\star}(K), \mathbf{u}^{\star}(K+1), \ldots, \mathbf{u}^{\star}(N-1)\right)$.

Proof: We assume that $\mathbf{u}^{\star, K}$ is not a POS of length $N-K$ for initial value $x^{\mathbf{u}^{\star}}(K, x)$. This implies the existence of $\mathbf{u} \in \mathbb{U}^{N-K}\left(x^{\mathbf{u}^{\star}}(K, x)\right)$ satisfying

$$
\begin{aligned}
& \forall i: J_{i}^{N-K}\left(x^{\mathbf{u}^{\star}}(K, x), \mathbf{u}\right) \leq J_{i}^{N-K}\left(x^{\mathbf{u}^{\star}}(K, x), \mathbf{u}^{\star, K}\right) \text { and } \\
& \exists j: J_{j}^{N-K}\left(x^{\mathbf{u}^{\star}}(K, x), \mathbf{u}\right)<J_{j}^{N-K}\left(x^{\mathbf{u}^{\star}}(K, x), \mathbf{u}^{\star, K}\right) .
\end{aligned}
$$

Hence,

$$
\begin{aligned}
\forall i: & J_{i}^{N}\left(x, \mathbf{u}^{\star}\right) \geq \\
& \sum_{k=0}^{K-1} \ell_{i}\left(x^{\mathbf{u}^{\star}}(k, x), u^{\star}(k)\right)+J_{i}^{N-K}\left(x^{\mathbf{u}^{\star}}(K, x), \mathbf{u}\right) \text { and } \\
\exists j: & J_{j}^{N}\left(x, \mathbf{u}^{\star}\right)= \\
& \sum_{k=0}^{K-1} \ell_{j}\left(x^{\mathbf{u}^{\star}}(k, x), u^{\star}(k)\right)+J_{j}^{N-K}\left(x^{\mathbf{u}^{\star}}(K, x), \mathbf{u}^{\star, K}\right) \\
> & \sum_{k=0}^{K-1} \ell_{j}\left(x^{\mathbf{u}^{\star}}(k, x), u^{\star}(k)\right)+J_{j}^{N-K}\left(x^{\mathbf{u}^{\star}}(K, x), \mathbf{u}\right) .
\end{aligned}
$$

This contradicts the fact that $\mathbf{u}^{\star} \in \mathbb{U}_{\mathcal{P}}^{N}(x)$.

\section{A Multiobjective MPC Algorithm}

In the sequel we will deal with the following question: Provided that we achieve a Pareto optimal control in every step of the MPC iteration, does the performance of the closed-loop solution inherit similar optimality properties from the iterations? We start our investigations under the following assumptions, which are a straightforward extension of the assumptions for single-objective MPC with terminal conditions, see also [2].

Assumption 3: 1) There is an equilibrium pair $\left(x_{*}, u_{*}\right) \in \mathbb{X} \times \mathbb{U}$, i.e., $f\left(x_{*}, u_{*}\right)=x_{*}$.

2) There are $\alpha_{\ell, i} \in \mathcal{K}$ such that all stage costs $\ell_{i}$, $i \in\{1, \ldots, s\}$, satisfy $\min _{u \in \mathbb{U}} \ell_{i}(x, u) \geq \alpha_{\ell, i}(\| x-$ $\left.x_{*} \|\right) \forall x \in \mathbb{X}$.

Assumption 4 (Lyapunov function terminal cost): We assume that $x_{*}$ from Assumption 3 is contained in $\mathbb{X}_{0}, F_{i}(x) \geq$ 0 for all $i$ and all $x \in \mathbb{X}_{0}$, and the existence of a local feedback $\kappa: \mathbb{X}_{0} \rightarrow \mathbb{U}$ satisfying

1) $f(x, \kappa(x)) \in \mathbb{X}_{0}$ and

2) $\forall x \in \mathbb{X}_{0}, \quad i \in\{1, \ldots, s\}: F_{i}(f(x, \kappa(x)))+$ $\ell_{i}(x, \kappa(x)) \leq F_{i}(x)$.

Imposing Assumption 4 ensures that it is always possible to remain within the terminal constraint set $\mathbb{X}_{0}$ and that the cost of this control action is bounded from above by the original terminal cost. As we will see in the proof of Theorem 11 this leads to the fact, that the terminal constraint $x(N) \in \mathbb{X}_{0}$ 
is more restrictive for smaller than for larger optimization horizon $N$. The following is the MO MPC algorithm we propose in this paper.

Algorithm 5 (MO MPC with terminal conditions):

(0) At time $n=0$ : Measure $x(n)$ and choose a POS $\mathbf{u}_{x(n)}^{\star, N} \in \mathbb{U}_{\mathcal{P}}^{N}(x(n))$. Go to (2).

(1) Measure $x(n)$. Choose a POS $\mathbf{u}_{x(n)}^{\star, N}$ such that

$$
J_{i}^{N}\left(x(n), \mathbf{u}_{x(n)}^{\star, N}\right) \leq J_{i}^{N}\left(x(n), \mathbf{u}_{x(n)}^{N}\right) \quad \forall i .
$$

(2) For $x:=x^{\mathbf{u}_{x(n)}^{\star, N}}(N, x(n))$ set

$$
\mathbf{u}_{x(n+1)}^{N}:=\left(u_{x(n)}^{\star, N}(1), \ldots, u_{x(n)}^{\star, N}(N-1), \kappa(x)\right) .
$$

(3) Apply the feedback $\mu^{N}(x(n)):=u_{x(n)}^{\star, N}(0)$, set $n=n+1$ and go to (1).

In the remainder of this section we analyze the feasibility of the choice of the POS $\mathbf{u}_{x(n)}^{\star, N} \in \mathbb{U}_{\mathcal{P}}^{N}(x(n))$ in Algorithm 5, step (1). The following Definitions 6, 7 and Theorem 8 from the theory of multiobjective optimization are adapted from [4], [15] to our setting. Theorem 8 implies the desired feasibility result. The idea is to find conditions which imply that there are nondominated points in the lower left part of $\mathcal{J}^{N}(x)$ (cf. Fig. 1). Finally, Lemma 9 gives easily checkable sufficient conditions for the assumptions of Theorem 8 .

Definition 6 (External stability): If the set of admissible values $\mathcal{J}^{N}(x), x \in \mathbb{X}_{N}$, has the property that for each $j \in$ $\mathcal{J}^{N}(x) \backslash \mathcal{J}_{\mathcal{P}}^{N}(x)$ there is $j_{\mathcal{P}} \in \mathcal{J}_{\mathcal{P}}^{N}(x)$ such that $j \geq j_{\mathcal{P}}$ holds componentwise, we call $\mathcal{J}^{N}(x)$ externally stable.

Definition 7 (Cone-Compactness): The set $\mathcal{J}^{N}(x)$ is called $\mathbb{R}_{\geq 0}^{s}$-compact if $\forall j \in \mathcal{J}^{N}(x)$ the set $\left(j-\mathbb{R}_{\geq 0}^{s}\right) \cap$ $\mathcal{J}^{N}(x)$ is compact.

Theorem 8: Given a horizon $N \in \mathbb{N}_{\geq 1}$ and an initial value $x \in \mathbb{X}_{N}$. If

1) $\mathcal{J}^{N}(x) \neq \emptyset$ and

2) $\mathcal{J}^{N}(x)$ is $\mathbb{R}_{\geq 0}^{s}$-compact,

then the set $\mathcal{J}^{N}(x)$ is externally stable.

We will now present sufficient conditions which ascertain that the requirements of Theorem 8 are met and which are fulfilled by the example in Section V.

Lemma 9: If $\mathbb{U}$ is compact, $\mathbb{X}$ and $\mathbb{X}_{0}$ are closed and $f, F_{i}$ and $\ell_{i}$ are continuous for all $i$, then the conditions of Theorem 8 are fulfilled for all $x \in \mathbb{X}_{N}$ and all $N \in \mathbb{N}$.

Proof: Let an initial value $x \in \mathbb{X}_{N}$ and a horizon $N \in \mathbb{N}_{\geq 1}$ be given.

1) It follows from the definition of $\mathbb{X}_{N}$ that that $\mathbb{U}^{N}(x) \neq$ $\emptyset$ for $x \in \mathbb{X}_{N}$ and therefore $\mathcal{J}^{N}(x) \neq \emptyset$.

2) It was proven in [3] that (under the given assumptions) the set $\Delta$, that contains all feasible trajectories with respective control sequences $\left(x^{\mathbf{u}}(\cdot), \mathbf{u}\right)$, is a compact subset of $Z:=\underbrace{\mathbb{R}^{n} \times \cdots \times \mathbb{R}^{n}}_{N \text { times }} \times \underbrace{\mathbb{R}^{m} \times \cdots \times \mathbb{R}^{m}}_{N-1 \text { times }}$. If we interpret $J^{N}$ as a function that maps from $Z$ to
$\mathbb{R}^{s}$, then the compactness of $\mathcal{J}^{N}(x)$ can be concluded from compactness of $\Delta$ and continuity of the $\ell_{i}$ and $F_{i}$. The cone-compactness required in condition 2 of Theorem 8 is an immediate consequence from the stronger property of compactness.

\section{Properties of The MO MPC Algorithm - PERFORMANCE AND CONVERGENCE}

Fig. 2 schematically visualizes the choice of the POS in step (1) of the algorithm. The bounds resulting from $\mathbf{u}_{x(n)}^{N}$ are visualized by the dashed lines and determine the set of nondominated points that may be chosen (thick, red line). The basic idea (formalized in Lemma 10) is that the control sequence $\mathbf{u}_{x(n)}^{N}$ in step (2) is a POS of length $N-1$ prolonged by the local feedback from Assumption 4 and that the prolongation reduces the value of the objective functions. Our preliminary considerations in Section III moreover show

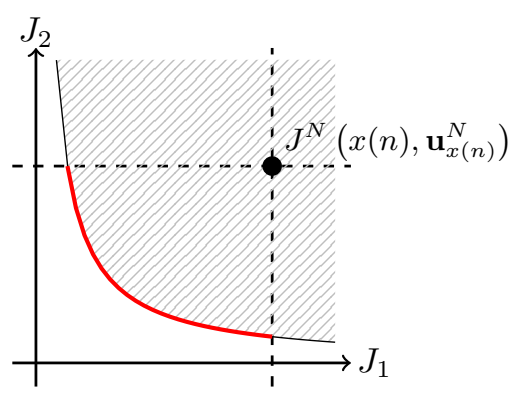

Fig. 2. Step (1) in Algorithm 5.

that - under appropriate assumptions - there is a POS with smaller objective value than the prolonged sequence (for each $i$ ). Hence, for each $\mathbf{u}^{\star, N-1} \in \mathbb{U}_{\mathcal{P}}^{N-1}(x)$ there is $\mathbf{u}^{\star, N} \in \mathbb{U}_{\mathcal{P}}^{N}(x)$ with

$$
\mathcal{J}_{i}^{N}\left(x, \mathbf{u}^{\star, N}\right) \leq J_{i}^{N-1}\left(x, \mathbf{u}^{\star, N-1}\right) \forall i \in\{1, \ldots, s\}
$$

Lemma 10: If Assumption 4 holds and if there is a POS $\mathbf{u}^{\star, N-1} \in \mathbb{U}_{\mathcal{P}}^{N-1}(x), x \in \mathbb{X}_{N}$, then there exists a sequence $\mathbf{u}^{N} \in \mathbb{U}^{N}(x)$ satisfying

$$
J_{i}^{N}\left(x, \mathbf{u}^{N}\right) \leq J_{i}^{N-1}\left(x, \mathbf{u}^{\star, N-1}\right) \quad \forall i \in\{1, \ldots, s\} .
$$

Proof: We define $\mathbf{u}^{N}$ as follows. $u^{N}(k):=u^{\star, N-1}(k)$ for $k=0, \ldots, N-2$ and $u^{N}(N-1):=\kappa(\bar{x})$ from Assumption 4, where $\bar{x}:=x^{\mathbf{u}^{N}}(N-1, x)$. Then $\mathbf{u}^{N}$ is feasible because $\mathbf{u}^{\star, N-1} \in \mathbb{U}^{N-1}(x)$, and therefore, $\bar{x} \in \mathbb{X}_{0}$. Assumption 4 ensures feasibility of $\kappa(\bar{x})$ and $f(\bar{x}, \kappa(\bar{x}))$. 
With the definition of $\mathbf{u}^{N}$ we obtain the estimates

$$
\begin{aligned}
J_{i}^{N}\left(x, \mathbf{u}^{N}\right)= & \sum_{k=0}^{N-1} \ell_{i}\left(x^{\mathbf{u}^{N}}(k, x), \mathbf{u}^{N}(k)\right)+F_{i}\left(x^{\mathbf{u}^{N}}(N, x)\right) \\
= & \sum_{k=0}^{N-2} \ell_{i}\left(x^{\mathbf{u}^{N}}(k, x), \mathbf{u}^{N}(k)\right)+\ell_{i}(\bar{x}, \kappa(\bar{x})) \\
& \quad+F_{i}\left(f_{i}(\bar{x}, \kappa(\bar{x}))\right) \\
\leq & \sum_{k=0}^{N-2} \ell_{i}\left(x^{\mathbf{u}^{\star, N-1}}(k, x), \mathbf{u}^{\star, N-1}(k)\right)+F_{i}(\bar{x}) \\
= & J_{i}^{N-1}\left(x, \mathbf{u}^{\star, N-1}\right) .
\end{aligned}
$$

By means of the ideas that we explained in the beginning of the section, we are now ready to give our main result on the performance of the MPC feedback on an infinite horizon.

Theorem 11 (MO MPC Performance Theorem):

Consider a problem with system dynamics (1), cost criteria (2), and let $N \in \mathbb{N}_{\geq 2}$. Let Assumptions 3 and 4 hold and let the set $\mathcal{J}^{N}(x)$ be externally stable for each $x \in \mathbb{X}_{N}$. Then, the MPC feedback $\mu^{N}: \mathbb{X} \rightarrow \mathbb{U}$ defined in Algorithm 5 has the following infinite-horizon closed-loop performance:

$$
\begin{aligned}
J_{i}^{\infty}\left(x(0), \mu^{N}\right) & :=\lim _{K \rightarrow \infty} \sum_{k=0}^{K-1} \ell_{i}\left(x(k), \mu^{N}(x(k))\right) \\
& \leq J_{i}^{N}\left(x(0), \mathbf{u}_{x(0)}^{\star, N}\right)
\end{aligned}
$$

for all objectives $i \in\{1, \ldots, s\}$, in which $\mathbf{u}_{x(0)}^{\star, N}$ denotes the POS of step (0) in Algorithm 5.

Proof: Feasibility: The existence of the POS in step (0) and (1) is concluded from external stability of $\mathcal{J}^{N}(x)$. Feasibility of $\mathbf{u}_{x(n+1)}^{N}$ in (2) follows from Assumption 4.

Performance: It follows from the definition of the cost functional in (2) that

$$
\begin{aligned}
J_{i}^{N}\left(x(k), \mathbf{u}_{x(k)}^{\star, N}\right) & =\ell_{i}\left(x(k), u_{x(k)}^{\star, N}(0)\right) \\
+ & J_{i}^{N-1}\left(f\left(x(k), u_{x(k)}^{\star, N}(0)\right), \mathbf{u}_{x(k)}^{\star, N}(\cdot+1)\right),
\end{aligned}
$$

and hence, for arbitrary $K \in \mathbb{N}_{\geq 1}$

$$
\begin{aligned}
& \sum_{k=0}^{K-1} \ell_{i}\left(x(k), \mu^{N}(x(k))\right)=\sum_{k=0}^{K-1} \ell_{i}\left(x(k), u_{x(k)}^{\star, N}(0)\right) \\
= & \sum_{k=0}^{K-1}\left[J_{i}^{N}\left(x(k), \mathbf{u}_{x(k)}^{\star, N}\right)\right. \\
& \left.\quad-J_{i}^{N-1}\left(f\left(x(k), u_{x(k)}^{\star, N}(0)\right), \mathbf{u}_{x(k)}^{\star, N}(\cdot+1)\right)\right] \\
\leq & \sum_{k=0}^{K-1}\left[J_{i}^{N}\left(x(k), \mathbf{u}_{x(k)}^{\star, N}\right)\right. \\
& \left.\quad-J_{i}^{N}\left(f\left(x(k), u_{x(k)}^{\star, N}(0)\right), \mathbf{u}_{x(k+1)}^{N}\right)\right],
\end{aligned}
$$

in which the inequality follows from Lemma 10 in combination with Lemma 2 , and $\mathbf{u}_{x(k)}^{\star, N}$ is the POS chosen in the algorithm at time $k$. In step (1), $\mathbf{u}_{x(k+1)}^{\star, N}$ is constructed such that

$$
J_{i}^{N}\left(x(k+1), \mathbf{u}_{x(k+1)}^{\star, N}\right) \leq J_{i}^{N}\left(x(k+1), \mathbf{u}_{x(k+1)}^{N}\right) .
$$

Thus, we finally obtain

$$
\begin{aligned}
& \sum_{k=0}^{K-1} \ell_{i}\left(x(k), \mu^{N}(x(k))\right) \\
\leq & J_{i}^{N}\left(x(0), \mathbf{u}_{x(0)}^{\star, N}\right)-J_{i}^{N}\left(x(K), \mathbf{u}_{x(K)}^{N}\right) \\
\leq & J_{i}^{N}\left(x(0), \mathbf{u}_{x(0)}^{\star, N}\right),
\end{aligned}
$$

because of the positivity of $J_{i}^{N}$. The expression on the left hand side of the inequality is monotonically increasing and due to its boundedness, the limit for $K \rightarrow \infty$ exists and we conclude the assertion.

Remark 12: A closer look at Algorithm 5 reveals that only for $k \geq 1$ the choice of $\mathbf{u}_{x(k)}^{\star, N}$ is subject to additional constraints (see step (1)). The first POS $\mathbf{u}_{x(0)}^{\star, N}$, which determines the bound on the performance of the MPC algorithm, can be chosen arbitrarily. Thus, the performance can be calculated a priori from a multiobjective optimization of horizon $N$.

Corollary 13: Under the assumptions of Theorem 11 it holds that the trajectory $x(\cdot)$ driven by the feedback $\mu^{N}$ from Algorithm 5 converges to the equilibrium $x_{*}$.

Proof: It follows from Theorem 11 that the sum $\quad \sum_{k=0}^{\infty} \ell_{i}\left(x(k), \mu^{N}(x(k))\right) \quad$ converges for each $i \in\{1, \ldots, s\}$. Hence, the sequences $\left(\ell_{i}\left(x(k), \mu^{N}(x(k))\right)\right)_{k \in \mathbb{N}_{0}}, i \in\{1, \ldots, s\}$, tend to zero. Together with Assumption 3 for arbitrary $i$ we obtain

$$
\begin{aligned}
\forall \varepsilon>0 & \exists K \in \mathbb{N}_{0}: \forall k \geq K: \\
\varepsilon & >\left|\ell_{i}\left(x(k), \mu^{N}(x(k))\right)\right|=\ell_{i}\left(x(k), \mu^{N}(x(k))\right) \\
& \geq \min _{u \in \mathbb{U}(x(k))} \ell_{i}(x(k), u) \geq \alpha_{\ell, i}\left(\left\|x(k)-x_{*}\right\|\right) .
\end{aligned}
$$

Since $\alpha_{\ell, i}$ is a $\mathcal{K}$ function, we conclude

$$
\begin{aligned}
\alpha_{\ell, i}\left(\lim _{k \rightarrow \infty}\left\|x(k)-x_{*}\right\|\right) & =\lim _{k \rightarrow \infty} \alpha_{\ell, i}\left(\left\|x(k)-x_{*}\right\|\right)=0 \\
\Leftrightarrow \lim _{k \rightarrow \infty}\left\|x(k)-x_{*}\right\| & =0 .
\end{aligned}
$$

We have proved in Theorem 11 that the inequalities

$$
J_{i}^{\infty}\left(x(0), \mu^{N}\right) \leq J_{i}^{N}\left(x(0), \mathbf{u}_{x(0)}^{\star, N}\right) \quad \forall i \in\{1, \ldots, s\}
$$

hold for the MPC feedback $\mu^{N}$ from Algorithm 5. Usually, one would like to compare the infinite-horizon MPC cost to an expression of the form $J_{i}^{\infty}\left(x(0), \mathbf{u}_{x(0)}^{\star, \infty}\right)$, where $\mathbf{u}_{x(0)}^{\star, \infty}$ is a POS for the infinite-horizon problem

$$
\begin{array}{ll}
\min _{\mathbf{u}} & \left(J_{1}^{\infty}(x(0), \mathbf{u}), \ldots, J_{s}^{\infty}(x(0), \mathbf{u})\right), \\
\text { with } & J_{i}^{\infty}(x(0), \mathbf{u}):=\sum_{k=0}^{\infty} \ell_{i}(x(k), u(k)) \\
\text { s.t. } \quad & x(k+1)=f(x(k), u(k)), \quad k \in \mathbb{N}_{0}, \\
& x(k) \in \mathbb{X}, k \in \mathbb{N} \\
& \mathbf{u} \in \mathbb{U}^{\infty} .
\end{array}
$$


We now show that it is, in general, not possible to bound $J_{i}^{\infty}\left(x(0), \mu^{N}\right)$ from above by $J_{i}^{\infty}\left(x(0), \mathbf{u}_{x(0)}^{\star, \infty}\right)$. Again, we summarize all constraints in (6) by writing $\mathbf{u} \in \mathbb{U}^{\infty}(x(0))$.

Lemma 14: Let $N \in \mathbb{N}_{\geq 2}, x \in \mathbb{X}_{N}$ be given. Let the assumptions of Theorem 11 hold and assume furthermore external stability of the set $\mathcal{J}^{\infty}(x):=$ $\left\{\left(J_{1}^{\infty}(x, \mathbf{u}), \ldots, J_{s}^{\infty}(x, \mathbf{u})\right) \mid \mathbf{u} \in \mathbb{U}^{\infty}(x)\right\}$. Then, for each $\mathbf{u}^{\star, N} \in \mathbb{U}_{\mathcal{P}}^{N}(x)$ there is $\mathbf{u}^{\star, \infty} \in \mathbb{U}_{\mathcal{P}}^{\infty}(x)$ such that the inequalities

$$
J_{i}^{N}\left(x, \mathbf{u}^{\star, N}\right) \geq J_{i}^{\infty}\left(x, \mathbf{u}^{\star, \infty}\right)
$$

hold for all $i=1, \ldots, s$.

Proof: For $N \in \mathbb{N}_{\geq 2}$ and $x \in \mathbb{X}_{N}$ fix an arbitrary $\mathbf{u}^{\star, N} \in \mathbb{U}_{\mathcal{P}}^{N}(x)$. Define the MPC feedback $\mu^{N}$ according to Algorithm 5 and define $\mathbf{u} \in \mathbb{U}^{\infty}(x)$ via $u(k)=\mu^{N}\left(x^{\mu^{N}}(k)\right)$ for $k \in \mathbb{N}_{\geq 0}$. Then, we have

$$
J_{i}^{N}\left(x, \mathbf{u}^{\star, N}\right) \stackrel{\text { Thm. } 11}{\geq} J_{i}^{\infty}\left(x, \mu^{N}\right)=J_{i}^{\infty}(x, \mathbf{u}) \quad \forall i .
$$

Since we assume external stability of the set $\mathcal{J}^{\infty}(x)$, there exists $\mathbf{u}^{\star, \infty} \in \mathbb{U}_{\mathcal{P}}^{\infty}(x)$ satisfying

$$
J_{i}^{\infty}(x, \mathbf{u}) \geq J_{i}^{\infty}\left(x, \mathbf{u}^{\star, \infty}\right) \quad \forall i .
$$

This yields the assertion.

Lemma 14 implies that Theorem 11 cannot be used to establish the inequality $J_{i}^{\infty}\left(x(0), \mu^{N}\right) \leq J_{i}^{\infty}\left(x(0), \mathbf{u}^{\star, \infty}\right)$. However, we will be able to show an approximate estimate of this form in Theorem 16. As a preparation, we first show that the trajectory corresponding to any infinite-horizon control sequence with bounded objectives gets arbitrarily close to the equilibrium $x_{*}$ in a finite number of time steps.

Lemma 15: Let $\delta>0, x \in \mathbb{X}$ and $\mathbf{u}^{\infty} \in \mathbb{U}^{\infty}(x)$ be given. Under Assumption 3 and if there is $K \in \mathbb{R}_{\geq 0}$ satisfying

$$
J_{i}^{\infty}\left(x, \mathbf{u}^{\infty}\right) \leq K \quad \forall i \in\{1, \ldots, s\},
$$

then the index $\hat{k}:=\min \left\{k \in \mathbb{N}_{0} \mid x^{\mathbf{u}^{\infty}}(k) \in \mathcal{B}_{\delta}\left(x_{*}\right)\right\}$ fulfills $\hat{k} \leq \frac{K}{\min _{i} \alpha_{\ell, i}(\delta)}$. Here, $\mathcal{B}_{\delta}\left(x_{*}\right):=\left\{x \in \mathbb{X}:\left\|x-x_{*}\right\| \leq \delta\right\}$.

Proof: Assume $\hat{k}>\frac{K}{\min _{i} \alpha_{\ell, i}(\delta)}$, then it holds

$$
\begin{aligned}
J_{i}^{\infty}\left(x, \mathbf{u}^{\infty}\right) & =\sum_{k=0}^{\hat{k}-1} \ell_{i}\left(x(k), u^{\infty}(k)\right)+\sum_{k=\hat{k}}^{\infty} \ell_{i}\left(x(k), u^{\infty}(k)\right) \\
& \geq \sum_{k=0}^{\hat{k}-1} \alpha_{\ell, i}\left(\left\|x(k)-x_{*}\right\|\right) \\
& >\sum_{k=0}^{\hat{k}-1} \alpha_{\ell, i}(\delta)=\hat{k} \cdot \alpha_{\ell, i}(\delta)>K,
\end{aligned}
$$

contradicting the assumption.

Theorem 16: Consider the optimal control problem (4) with cost criteria (2) and the corresponding optimal control problem on the infinite horizon (6) with the same constraints and running costs. Let the Assumptions 3 and 4 hold and assume furthermore the existence of $\sigma_{i} \in \mathcal{K}$ such that $F_{i}(x) \leq \sigma_{i}\left(\left\|x-x_{*}\right\|\right)$ holds for all $x \in \mathbb{X}_{0}$ and all $i \in$ $\{1, \ldots, s\}$. Consider an arbitrary initial value $x \in \mathbb{X}_{N}$ and a sequence $\mathbf{u}^{\star, \infty} \in \mathbb{U}_{\mathcal{P}}^{\infty}(x)$ with $J_{i}^{\infty}\left(x, \mathbf{u}^{\star, \infty}\right) \leq C \forall i, C \in$ $\mathbb{R}_{\geq 0}$. Assume there is $\bar{N} \in \mathbb{N}$ such that the sets $\mathcal{J}^{N}(x)$ are externally stable for all $N \geq \bar{N}$. Then, for each $\varepsilon>0$ there exists $N_{0} \in \mathbb{N}$ such that for all $N \geq N_{0}$ there is $\mathbf{u}^{\star, N} \in \mathbb{U}_{\mathcal{P}}^{N}(x)$ satisfying

$$
J_{i}^{N}\left(x, \mathbf{u}^{\star, N}\right) \leq J_{i}^{\infty}\left(x, \mathbf{u}^{\star, \infty}\right)+\varepsilon \quad \forall i
$$

In particular, $\mathbf{u}^{\star, \infty}$ can be approximated arbitrarily well by $\mu^{N}$ in terms of the infinite-horizon performance, that is,

$$
J_{i}^{N}\left(x, \mu^{N}\right) \leq J_{i}^{\infty}\left(x, \mathbf{u}^{\star, \infty}\right)+\varepsilon .
$$

Proof: Let $\varepsilon>0$ and choose $\delta>0$ such that $\sigma_{i}(\delta) \leq$ $\varepsilon \forall i$ and $\mathcal{B}_{\delta}\left(x_{*}\right) \subseteq \mathbb{X}_{0}$. For the sequence $\mathbf{u}^{\star, \infty} \in \mathbb{U}_{\mathcal{P}}^{\infty}(x)$ it holds $J_{i}^{\infty}\left(x, \mathbf{u}^{\star, \infty}\right) \leq C \forall i$. From Lemma 15 we know that the index $\hat{k}:=\min \left\{k \in \mathbb{N}_{0} \mid x^{\mathbf{u}^{\star, \infty}}(k) \in \mathcal{B}_{\delta}\left(x_{*}\right)\right\}$ satisfies $\hat{k} \leq \frac{C}{\min _{i} \alpha_{\ell, i}(\delta)}$. Now let us choose $N_{0} \in \mathbb{N}$ such that $N_{0} \geq$ $\max \{\hat{k}+1, \bar{N}\}$. For $N \geq N_{0}$ define the sequence $\mathbf{u} \in$ $\mathbb{U}^{N}(x)$ via

$$
u(k)= \begin{cases}u^{\star, \infty}(k), & k=0, \ldots, \hat{k}-1 \\ \kappa(x(k)), & k=\hat{k}, \ldots, N-1\end{cases}
$$

with $\kappa$ from Assumption 4. Since $x^{\mathbf{u}^{\star, \infty}}(\hat{k}) \in \mathcal{B}_{\delta}\left(x_{*}\right) \subseteq$ $\mathbb{X}_{0}, \kappa$ can be applied and it holds $x^{\mathbf{u}}(N) \in \mathbb{X}_{0}$. From the definition of $\mathbf{u}$ we obtain

$$
\begin{aligned}
& J_{i}^{N}(x, \mathbf{u})=\sum_{k=0}^{N-1} \ell_{i}(x(k), u(k))+F_{i}(x(N)) \\
& \sum_{k=0}^{k-1} \ell_{i}\left(x(k), u^{\star, \infty}(k)\right)+\sum_{k=\hat{k}}^{N-1} \ell_{i}(x(k), \kappa(x(k))) \\
& \quad+F_{i}(x(N)) \\
\leq & J_{i}^{\infty}\left(x, \mathbf{u}^{\star, \infty}\right)+\sum_{k=\hat{k}}^{N-1}\left[F_{i}(x(k))-F_{i}(f(x(k), \kappa(x(k))))\right] \\
& \quad+F_{i}(x(N)) \\
= & J_{i}^{\infty}\left(x, \mathbf{u}^{\star, \infty}\right)+F_{i}(x(\hat{k})) \\
\leq & J_{i}^{\infty}\left(x, \mathbf{u}^{\star, \infty}\right)+\sigma_{i}(\underbrace{\left\|x(\hat{k})-x_{*}\right\|}_{\leq \delta}) \\
\leq & J_{i}^{\infty}\left(x, \mathbf{u}^{\star, \infty}\right)+\varepsilon .
\end{aligned}
$$

Due to external stability of $\mathcal{J}^{N}(x)$ we conclude the existence of $\mathbf{u}^{\star, N} \in \mathbb{U}_{\mathcal{P}}^{N}(x)$ such that

$$
J_{i}^{N}\left(x, \mathbf{u}^{\star, N}\right) \leq J_{i}^{N}(x, \mathbf{u}) \leq J_{i}^{\infty}\left(x, \mathbf{u}^{\star, \infty}\right)+\varepsilon,
$$

i.e. (7) holds. Choosing $\mathbf{u}_{x(n)}^{\star, N}=\mathbf{u}^{\star, N}$ in step (0) of Algorithm 5 and combining the estimates (5) and (7) yields (8). 


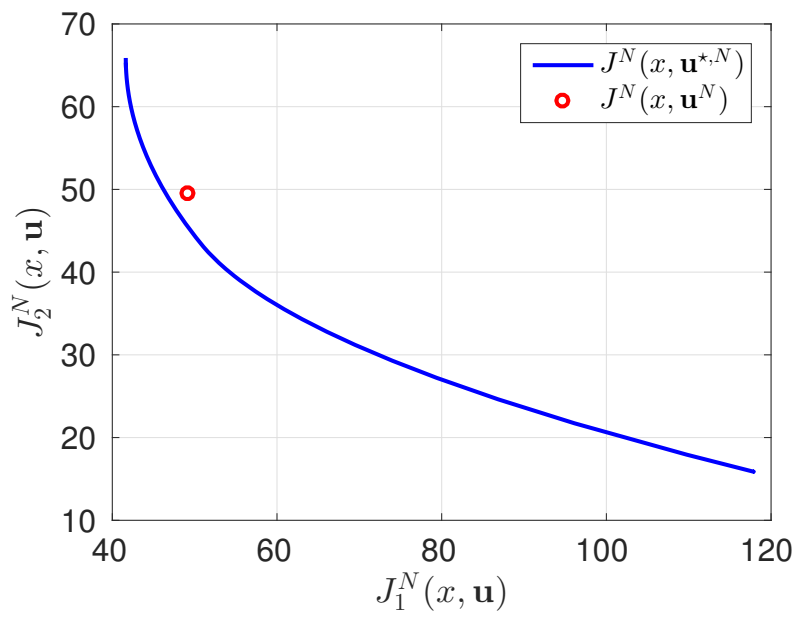

Fig. 3. Visualization of the Pareto front for Example 17, $N=2$ and $x=x(1)$ and the bounds in step (1) in the algorithm.

\section{NumERICAL EXAMPLE}

Example 17: We consider the following linear optimal control problem with two objectives from [2].

$$
\begin{aligned}
x^{+} & =A x+B u \\
\ell_{1}(x, u) & =x^{T} Q_{1} x+u^{T} R_{1} u, F_{1}(x)=x^{T} P_{1} x \\
\ell_{2}(x, u) & =\left\|Q_{2} x\right\|_{\infty}+\left\|R_{2} u\right\|_{\infty} F_{2}(x)=\left\|P_{2} x\right\|_{\infty}, \\
A & =\left(\begin{array}{cc}
1 & 1 \\
0 & 1
\end{array}\right), B=\left(\begin{array}{c}
0.5 \\
1
\end{array}\right), Q_{1}=\left(\begin{array}{cc}
0.1 & 0 \\
0 & 1
\end{array}\right), \\
R_{1} & =0.2, P_{1}=\left(\begin{array}{cc}
0.5649 & 0.4054 \\
0.4054 & 1.6027
\end{array}\right), \\
Q_{2} & =\left(\begin{array}{cc}
1 & 0 \\
0 & 0.1
\end{array}\right), R_{2}=0.1, \\
P_{2} & =\left(\begin{array}{cc}
9.6085 & 1.1401 \\
-0.2965 & 9.4107
\end{array}\right)
\end{aligned}
$$

and constraints $\left|x_{1}\right|,\left|x_{2}\right|,|u| \leq 10$. Assumption 4 is satisfied with a linear feedback and terminal region $\mathbb{X}_{0}$ (cf. [2]). Moreover, the example sastisfies the assumptions of Theorem 11. We have implemented Algorithm 5 in MATLAB to illustrate our theoretical findings. The convex MO optimization problems were solved using a weighted sum approach (see e.g. [4] for a proof that this yields POS). In Fig. 3 we see the example of a Pareto front for Example 17 and the value $J^{N}\left(x(1), \mathbf{u}_{x(1)}^{N}\right)$ that determines the possible choices for the POS in step (1) of the algorithm at time $n=1$. Fig. 4 shows that the infinite-horizon closed-loop cost $J_{i}^{\infty}\left(x, \mu^{N}\right)$ is bounded by $J_{i}^{N}\left(x, \mathbf{u}_{x}^{\star, N}\right)$, i.e., the cost of the first PO chosen in Algorithm 5. This confirms the statement of Theorem 11. Moreover, we see in Fig. 5 that the closed-loop trajectories converge to the origin as stated in Corollary 13 . To demonstrate the nessecity of the constraint in step (1) of Algorithm 5, we run the algorithm without this constraint. In Fig. 6 we see that the first objective violates the upper bound but still converges. Thus, the trajectories still converge (not illustrated) but the performance deteriorates.
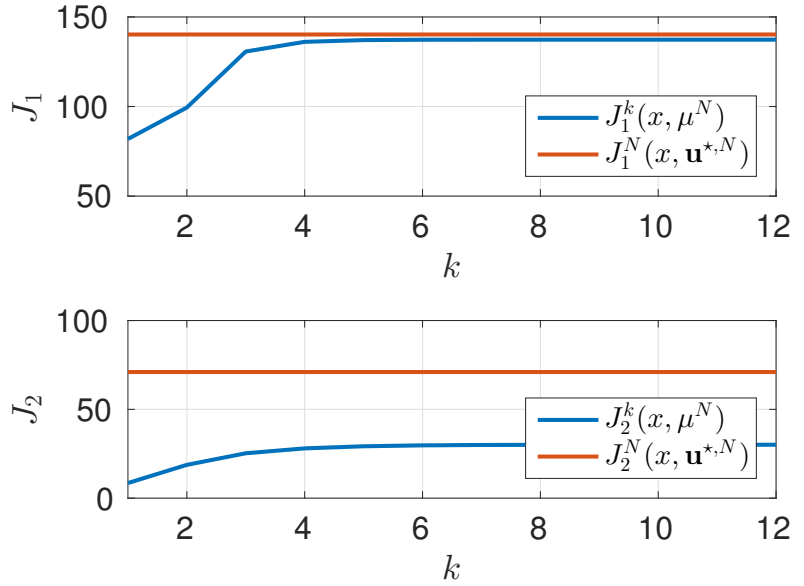

Fig. 4. Comparison of the accumulated MPC cost to the theoretically deduced upper bound for both objectives of Example 17 for $N=2$ and $x=x(0)=(7.5,7.5)^{T}$.

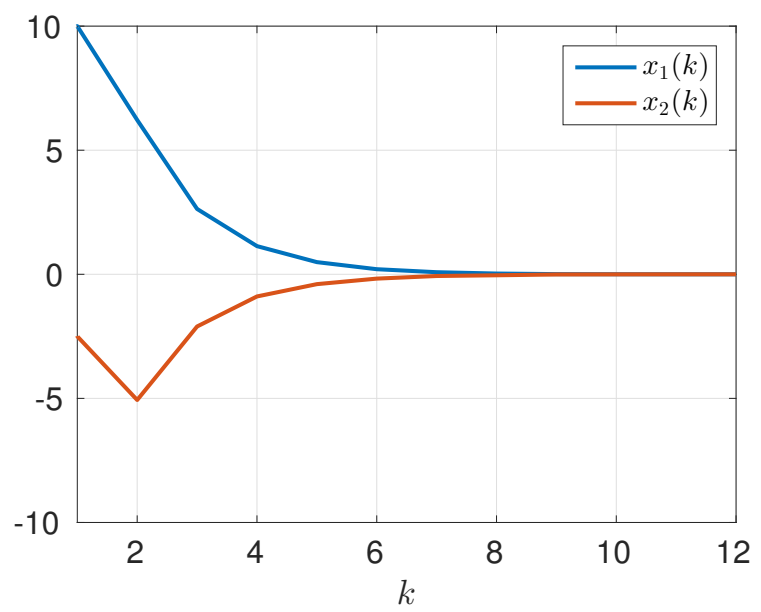

Fig. 5. Closed-loop trajectories of Example 17 for $N=2$ and $x(0)=$ $(7.5,7.5)^{T}$.
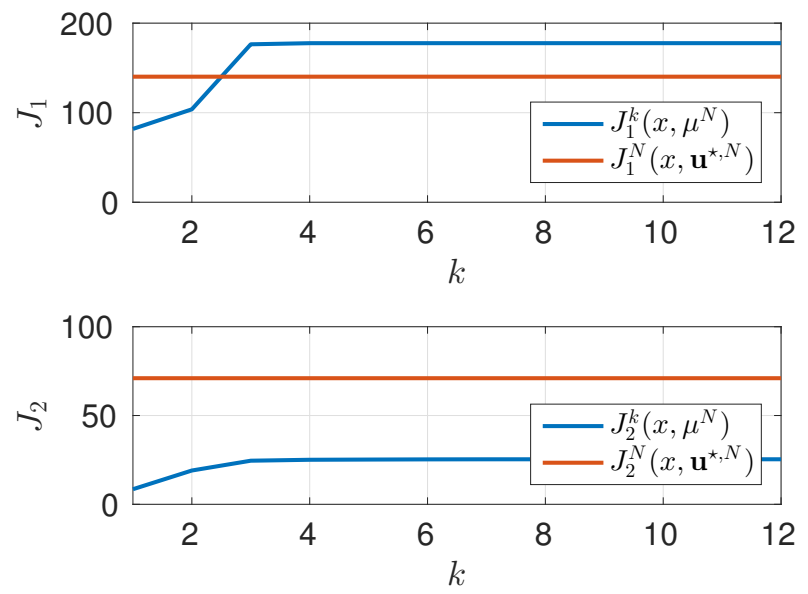

Fig. 6. Performance of the closed loop without the constraint in step (1) of Algorithm 5. 


\section{Multiobjective Economic MPC}

In case at least one of the cost criteria does not satisfy Assumption 3 but rather reflects some 'economic' objective, the theory presented in Section III does not apply to the control problem. As in the single-objective setting, it is still possible to make performance statements. The following assumption on the terminal condition, which is an extension of [1, Assumption 6] (see also [7, Assumption 8.5]), takes the place of the Assumptions 3 and 4 .

Assumption 18: 1) There is an equilibrium $\left(x_{*}, u_{*}\right) \in$ $\mathbb{X} \times \mathbb{U}$ with $x_{*} \in \mathbb{X}_{0}$ and $F_{i}\left(x_{*}\right)=0 \forall i \in\{1, \ldots, s\}$.

2) There is $\kappa: \mathbb{X}_{0} \rightarrow \mathbb{U}$ such that $f(x, \kappa(x)) \in \mathbb{X}_{0}$ and $\forall i \in\{1, \ldots, s\}$ it holds

$$
F_{i}(f(x, \kappa(x))) \leq F_{i}(x)-\ell_{i}(x, \kappa(x))+\ell_{i}\left(x_{*}, u_{*}\right) .
$$

By means of these conditions we can formulate an averaged performance result for Algorithm 5 for the economic setting.

Theorem 19 (Averaged Performance Theorem): Consider a problem with system dynamics (1), cost criteria (2), and let $N \in \mathbb{N}_{\geq 2}$. Let Assumption 18 hold and let the set $\mathcal{J}^{N}(x)$ be externally stable for each $x \in \mathbb{X}_{N}$. We furthermore assume, that there is $M \in \mathbb{R}$ such that $J_{i}^{N}\left(x, \mathbf{u}^{\star}\right) \geq M$ for all $x \in \mathbb{X}_{N}, \mathbf{u}^{\star} \in \mathbb{U}_{\mathcal{P}}^{N}(x)$ and $i \in\{1, \ldots, s\}$. Then, the MPC feedback $\mu^{N}: \mathbb{X} \rightarrow \mathbb{U}$ defined in Algorithm 5 has the following infinite-horizon averged closed-loop performance:

$$
\limsup _{K \rightarrow \infty} \frac{1}{K} \sum_{k=0}^{K-1} \ell_{i}\left(x(k), \mu^{N}(x(k))\right) \leq \ell_{i}\left(x_{*}, u_{*}\right) .
$$

Proof: We follow the reasoning in the proof of Theorem 11. Feasibility of all chosen POS holds with the same arguments. For the performance we obtain

$$
\begin{aligned}
& \sum_{k=0}^{K-1} \ell_{i}\left(x(k), \mu^{N}(x(k))\right) \\
= & \sum_{k=0}^{K-1}\left[J_{i}^{N}\left(x(k), \mathbf{u}_{x(k)}^{\star, N}\right)\right. \\
& \left.\quad-J_{i}^{N-1}\left(f\left(x(k), u_{x(k)}^{\star, N}(0)\right), \mathbf{u}_{x(k)}^{\star, N}(\cdot+1)\right)\right] \\
\leq & \sum_{k=0}^{K-1}\left[J_{i}^{N}\left(x(k), \mathbf{u}_{x(k)}^{\star, N}\right)\right. \\
& \left.\quad-J_{i}^{N}\left(f\left(x(k), u_{x(k)}^{\star, N}(0)\right), \mathbf{u}_{x(k+1)}^{N}\right)+\ell_{i}\left(x_{*}, u_{*}\right)\right],
\end{aligned}
$$

in which the inequality is obtained as in Lemma 10 for the terminal cost from Assumption 18 in combination with Lemma 2, and $\mathbf{u}_{x(k)}^{\star N}$ is the POS chosen in the algorithm at time $k$. In step (1) $\mathbf{u}_{x(k+1)}^{\star, N}$ is constructed such that

$$
J_{i}^{N}\left(x(k+1), \mathbf{u}_{x(k+1)}^{\star, N}\right) \leq J_{i}^{N}\left(x(k+1), \mathbf{u}_{x(k+1)}^{N}\right) .
$$

Thus, we obtain

$$
\begin{aligned}
& \sum_{k=0}^{K-1} \ell_{i}\left(x(k), \mu^{N}(x(k))\right) \\
\leq & J_{i}^{N}\left(x(0), \mathbf{u}_{x(0)}^{\star, N}\right)-J_{i}^{N}\left(x(K), \mathbf{u}_{x(K)}^{\star, N}\right)+K \ell_{i}\left(x_{*}, u_{*}\right) \\
\leq & J_{i}^{N}\left(x(0), \mathbf{u}_{x(0)}^{\star, N}\right)-M+K \ell_{i}\left(x_{*}, u_{*}\right) .
\end{aligned}
$$

Taking the average and the limit superior on both sides of the inequality yields the assertion.

As before, we are again able to estimate the performance of every single objective.

\section{CONCLUSIONS}

In this paper we have developed an MO MPC algorithm that allows for a performance analysis for all cost criteria and which does not rely on a specific multiobjective optimization method. Results for MO MPC without terminal conditions for the stabilizing as well as for the economic setting are subject of our current research.

\section{REFERENCES}

[1] R. Amrit, J. B. Rawlings, and D. Angeli, "Economic optimization using model predictive control with a terminal cost," Annual Rev. Control, vol. 35, pp. 178-186, 2011.

[2] A. Bemporad and D. Muñoz de la Peña, "Multiobjective model predictive control," Automatica, vol. 45, pp. 2823-2830, 2009.

[3] J. Doležal, "Existence of optimal solutions in general discrete systems," Kybernetika, vol. 11, no. 4, pp. 301-312, 1975.

[4] M. Ehrgott, Multicriteria Optimization, 2nd ed. Springer, 2005.

[5] J. J. V. García, V. G. Garay, E. I. Gordo, F. A. Fano, and M. L. Sukia, "Intelligent multi-objective nonlinear model predictive control (imonmpc): Towards the on-line optimization of highly complex control problems," Expert systems with applications, vol. 39, no. 7, pp. 65276540, 2012

[6] P. Giselsson and A. Rantzer, "Distributed Model Predictive Control with Suboptimality and Stability Guarantees," in 49th IEEE Conference on Decision and Control (CDC). IEEE, 2010, pp. 7272-7277.

[7] L. Grüne and J. Pannek, Nonlinear Model Predictive Control: Theory and Algorithms, 2nd ed., ser. Communications and Control Engineering. Springer, 2017.

[8] C. M. Hackl, F. Larcher, A. Dötlinger, and R. M. Kennel, "Is multiple-objective model-predictive control "optimal"?" in 2013 IEEE International Symposium on Sensorless Control for Electrical Drives and Predictive Control of Electrical Drives and Power Electronics (SLED/PRECEDE), 2013.

[9] D. He, L. Wang, and J. Sun, "On stability of multiobjective NMPC with objective prioritization," Automatica, vol. 57, pp. 189-198, 2015.

[10] K. Laabidi, F. Bouani, and M. Ksouri, "Multi-criteria optimization in nonlinear predictive control," Mathematics and Computers in Simulation, vol. 76, no. 56, pp. 363-374, 2008.

[11] J. Maestre, D. Muñoz de la Peña, and E. Camacho, "Distributed model predictive control based on a cooperative game," Optimal Control Applications and Methods, vol. 32, no. 2, pp. 153-176, 2011.

[12] H. Nakayama, Y. Yun, and M. Shirakawa, "Multi-objective model predictive control," in Multiple Criteria Decision Making for Sustainable Energy and Transportation Systems, M. Ehrgott, B. Naujoks, T. Stewart, and J. Wallenius, Eds. Springer, 2010, pp. 277-287.

[13] A. Núñez, C. E. Cortés, D. Sáez, B. De Schutter, and M. Gendreau, "Multiobjective model predictive control for dynamic pickup and delivery problems," Control Engineering Practice, vol. 32, pp. 7386, 2014

[14] J. B. Rawlings and D. Q. Mayne, Model Predictive Control: Theory and Design. Nob Hill Publishing, 2009.

[15] Y. Sawaragi, H. Nakayama, and T. Tanino, Theory of multiobjective optimization. Elsevier, 1985.

[16] B. T. Stewart, A. N. Venkat, J. B. Rawlings, S. J. Wright, and G. Pannocchia, "Cooperative distributed model predictive control," Systems \& Control Letters, vol. 59, no. 8, pp. 460-469, 2010.

[17] M. Vallerio, J. Van Impe, and F. Logist, "Tuning of NMPC controllers via multi-objective optimisation," Computers \& Chemical Engineering, vol. 61, pp. 38-50, 2014.

[18] V. M. Zavala and A. Flores-Tlacuahuac, "Stability of multiobjective predictive control: A utopia-tracking approach," Automatica, vol. 48, no. 10, pp. 2627-2632, 2012. 